\title{
COLD BUBBLE FORMATION DURING TOKAMAK DENSITY LIMIT DISRUPTIONS
}

\author{
J. HOWARD, M. PERSSON* \\ Plasma Research Laboratory, \\ Research School of Physical Sciences, \\ Australian National University, \\ Canberra, Australia
}

\begin{abstract}
Tokamak density limit disruptions are studied by tomographically analysing soft X-ray emission data using a modified circular harmonic reconstruction technique. Both minor and major disruptions are associated with the presence of dominant $\mathrm{m}=1 / \mathrm{n}=1$ and $\mathrm{m}=2 / \mathrm{n}=1$ soft X-ray emissivity perturbations leading to the penetration of a cold bubble-like plasma volume to the centre. The role of mode coupling between the various emission components is examined and the observed behaviour is compared with the results of recent theoretical models.
\end{abstract}

\section{INTRODUCTION}

The operational regime of tokamaks is limited with respect to current and particle density. The experimentally observed density limit for an ohmically heated plasma can be expressed as $n<\mathrm{CB}_{\mathrm{T}} / \mathrm{Rq}_{\mathrm{a}}$, where $\mathrm{B}_{\mathrm{T}}$ is the toroidal magnetic field, $R$ is the major radius, $\mathrm{q}_{\mathrm{a}}$ is the safety factor at the plasma edge and $\mathrm{C}$ is of the order of $(1-2) \times 10^{20} \mathrm{~Wb}^{-1}[1-5]$. If this limit is exceeded, the discharge suffers a major disruption which leads to loss of confinement and a sudden termination of the plasma. Often, this major disruption is preceded by a series of minor disruptions from which the plasma recovers. Recently, extended and softer density limits have been obtained with beryllium in belt limiters and beryllium coating of the vacuum vessel in the JET tokamak [6].

Density limit disruptions have been associated with the cooling of the outer part of the plasma and it has been shown that the limit in $\mathrm{nRq}_{\mathrm{a}} / \mathrm{B}_{\mathrm{T}}$ is compatible with a $100 \%$ power loss by radiation at a fixed impurity level $[7,8]$. With the energy mainly lost through radiation, the plasma becomes thermally detached from the wall, which leads to a narrowing of the temperature and current profiles as the radiation front propagates inwards. This can result in a current profile with only a small amount of current flowing outside the $q=2$ surface, which in turn has a destabilizing influence on the $2 / 1$ tearing mode [9] - a major player in the disruption process.

\footnotetext{
* Also at Department of Theoretical Physics.
}

However, while the initial contracting phase of density limit disruptions appears to be relatively well understood $[4,7,8]$, the further development is not so clear. With $\mathrm{q}_{0} \sim 1$, the $2 / 1$ mode saturates at a reasonably small magnetic island width [9] and it appears that one or more additional mechanisms must be responsible for a further destabilization of the plasma. One such mechanism is wall locking, which takes place at the $q$ limit $[10,4]$ as well as before many density limit disruptions [11]. At high-q operation, locking of the modes to the wall is particularly destabilizing for profiles with most of the current inside the $q=2$ surface [12]. An alternative scenario for destabilization of the plasma is suggested by the recent simulations of Bondeson et al. [13]. In their model, an inward propagating radiation front destabilizes the low-order Fourier modes; in particular, the $2 / 1$ mode grows to a high level. A broad spectrum of modes are linearly unstable to the quasi-equilibrium created by the $2 / 1$ mode, and non-linear coupling and growth of these components leads to a large region of field line stochasticity. This broadens the current profile and further destabilizes the $2 / 1$ mode. Another possible trigger mechanism for the disruption is the penetration to the plasma centre of a cold bubble from the cooler edge plasma, as proposed by Kleva et al. [14]. When the cold bubble penetrates the interior regions, a current profile with a double set of $q=2$ surfaces, which is violently unstable to the $2 / 1$ mode, can be created.

In this paper we report results from tomographic reconstructions of the soft X-ray (SXR) emission during a sequence of disruptions in the LT-4 tokamak. In 
particular, we examine the disruption mechanism and compare the results with recent numerical modelling. Many of the qualitative features of Ref. [13] are examined, including the coupling between the $1 / 1$ and $2 / 1$ modes and their $3 / 2$ and $-1 / 0$ side-bands, the fixed phase relationship between the modes, and the 'bubblelike' erosion of the plasma temperature (and hence SXR) profile. The cold bubble scenario of Ref. [14] is also considered in some detail.

The paper is organized as follows: The experimental geometry and the plasma conditions are described in Section 2. Also, the data analysis techniques are summarized; the novel tomographic approach is described in the Appendix. Reconstructed emission contours for the minor disruptions and experimental results are presented and compared with recent numerical simulations in Section 3. The behaviour during a major disruption is examined in Section 4 and the results are discussed in Section 5.

\section{EXPERIMENT AND DATA ANALYSIS}

The SXR apparatus in LT-4 [15] has been used for emission measurements on the LT-4 tokamak $[15,16]$
$\left(\mathrm{R}=0.5 \mathrm{~m}, \mathrm{a} \sim 0.10 \mathrm{~m}, \overline{\mathrm{n}}_{\mathrm{e}} \sim 3 \times 10^{13} \mathrm{~cm}^{-3}\right.$, $B_{T} \sim 1-2 T, T_{e} \leqslant 500 \mathrm{eV}, I_{P} \leqslant 70 \mathrm{kA}$ ). The camera comprises 18 surface barrier diode detectors, viewing the plasma through a slit of a width of $1 \mathrm{~mm}$ about a horizontal axis through the centre and in a poloidal section of the plasma. The chords in the fan array have impact parameters separated by about $1 \mathrm{~cm}$, in the range of $8-9 \mathrm{~cm}$ about the nominal plasma centre (Fig. 1). The chord integrated emission is collected from a region of finite radial extent such that the fan projection is essentially band limited, slightly oversampled and hence alias free. The detectors respond to radiation in the range $1.2-10.4 \mathrm{keV}$ and are relatively calibrated to $\$ 1 \%$ accuracy. The signals are digitized at a $200 \mathrm{kHz}$ rate and the signal to noise ratio in the central channels is better than 100. A discussion of the SXR tomography is given in Refs $[17,18]$.

With reference to the toroidal co-ordinate system $(\mathrm{r}, \theta, \phi)$ shown in Fig. 1 , both $\mathrm{B}_{\mathrm{T}}$ and $\mathrm{I}_{\mathrm{P}}$ are in the negative toroidal direction. For convenience, we take $\phi=0$ at the position of the SXR diagnostic. A set of eight poloidally equispaced pick-up coils for monitoring the poloidal component of the MHD activity are installed in a cross-section displaced toroidally by $3^{\circ}$ from the SXR camera. The low-pass cut-off for this coil set is limited by the tokamak wall to $\sim 15 \mathrm{kHz}$. The coil

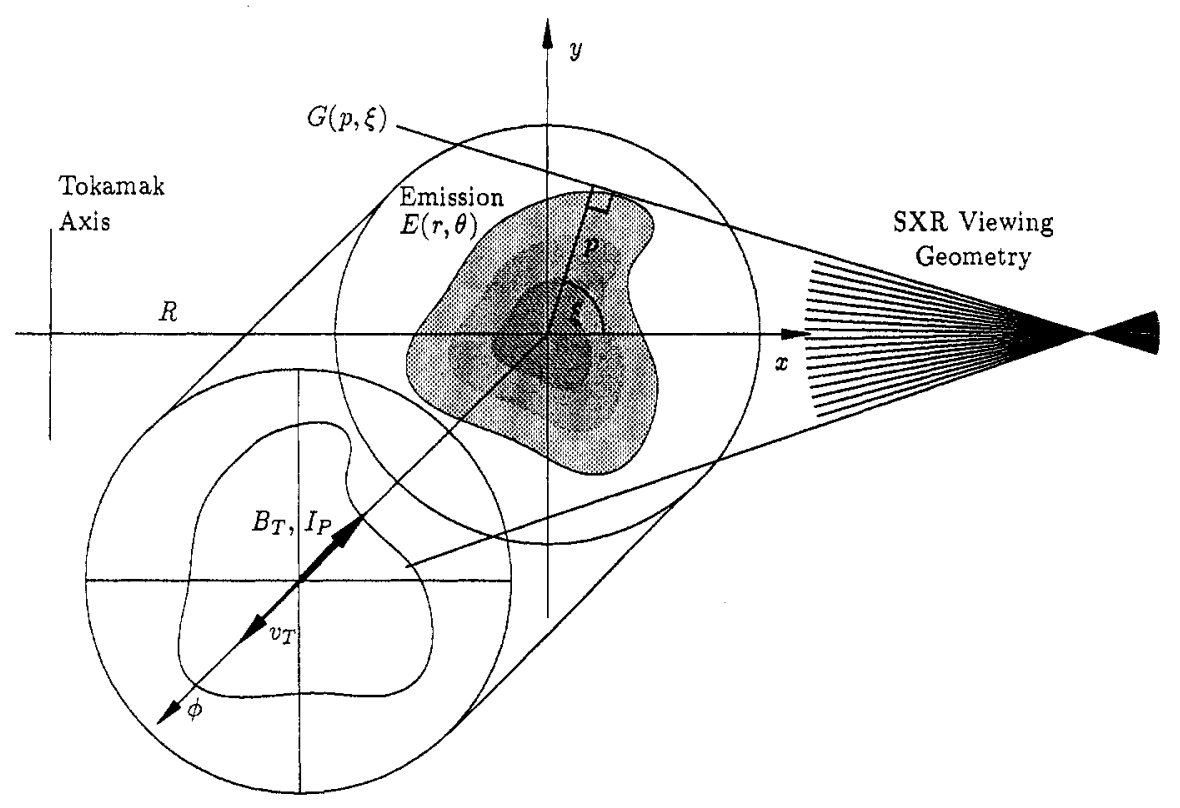

FIG. 1. Schematic diagram of the SXR measurement geometry on LT-4. Also shown are the projection co-ordinates $(p, \xi)$, the polar image co-ordinates $(r, \theta)$ and the directions of $B_{T}, I_{P}$ and $\mathrm{v}_{T}$ relative to the toroidal direction $\phi$. 

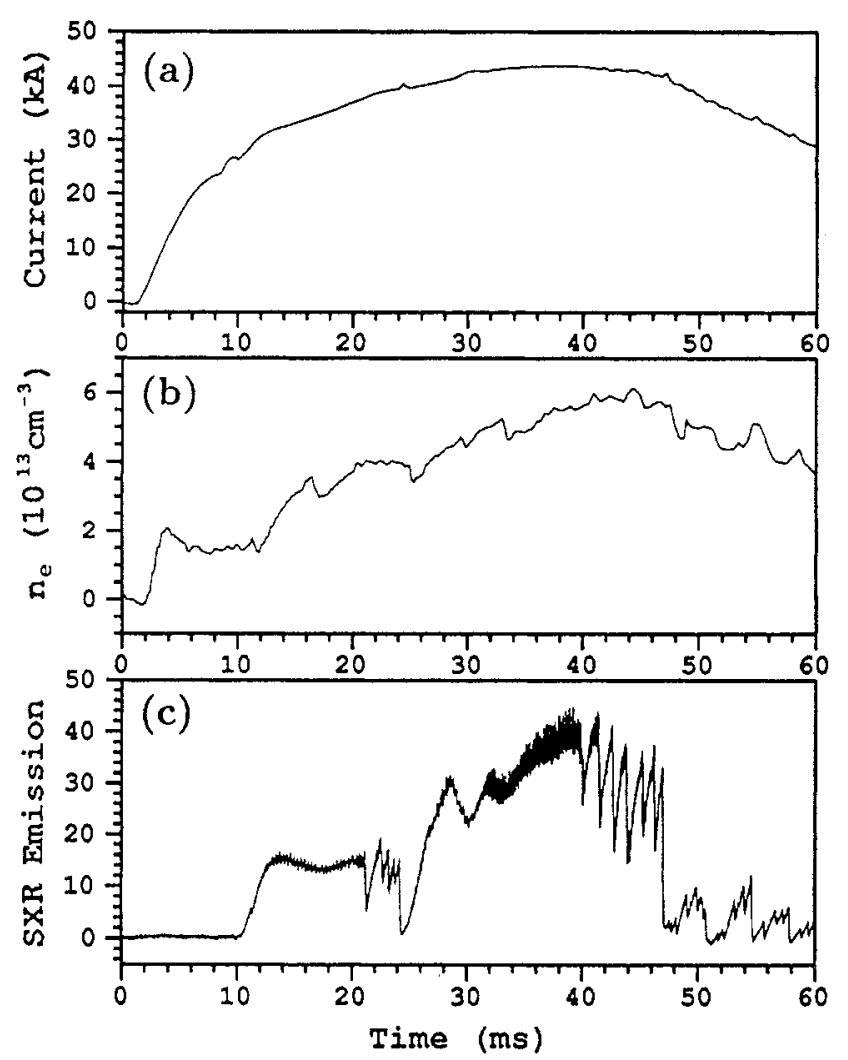

FIG. 2. Temporal evolution of (a) the plasma current, (b) the central line averaged density and (c) the central SXR emission. Note the sawtooth activity between 14 and $20 \mathrm{~ms}$ and the disruption sequence between 38 and $48 \mathrm{~ms}$.

signals presented here have been integrated and corrected for the wall time delay. The poloidal variation of the slope of the magnetic field lines due to toroidal curvature is, to first order, proportional to the inverse machine aspect ratio [19]. However, it has been confirmed by azimuthal analysis of the coil signals that because of the moderately large aspect ratio, $R / a \geq 5$, the coupling between the harmonics is negligible. In the following, these harmonics are identified with the corresponding MHD linear eigenmodes having the same poloidal $\mathrm{m}$ number. Given the right-handed helicity of the confining field, the clockwise rotation of the signals registered by the coil array indicates that the bulk toroidal rotation is in the positive $\phi$ direction (opposite to $\mathbf{B}_{\mathrm{T}}$ and $\mathrm{I}_{\mathrm{P}}$ ).

Figure 2 shows the plasma current, the line averaged electron density and the central SXR emission for LT-4 discharge No. 552482 during disruption activity. The SXR signals show a cascade of sawtooth-like minor disruptions (coexistent with a high level of MHD activity) which commence at $\mathrm{t} \approx 40.0 \mathrm{~ms}$ and terminate in a severe and sudden decrease in plasma emission at $\mathrm{t} \approx 47.0 \mathrm{~ms}$ (Fig. 2). The unusually fast and accurate position feedback control system on LT-4 ensures survival of the degraded plasma even after the major disruption event (see Fig. 2). It can be seen in Fig. 2(b) that the disruptions occur when the electron density is maximum. This discharge, with $\mathrm{B}_{\mathrm{T}}=1.45 \mathrm{~T}$ and $\mathrm{C}-\overrightarrow{\mathrm{n}}_{\mathrm{e}} \mathrm{Rq}_{\mathrm{a}} / \mathrm{B}_{\mathrm{T}} \sim 1 \times 10^{20} \mathrm{~Wb}^{-1}$, is at the high density limit in the Hugill operating space for LT-4 [15].

It is important to note the major differences between the disruption activity and normal LT-4 sawtooth behaviour. The impact parameter about which the disruption SXR signals invert is $r_{\text {inv }} \approx 0.6 a$ and the typical change in the central line integrated emission is $\Delta \mathrm{G} / \mathrm{G} \geq 0.5$. By way of contrast, standard sawteeth (visible between times $\mathrm{t}=14 \mathrm{~ms}$ and $\mathrm{t}=20 \mathrm{~ms}$ in Fig. 2) show $r_{\text {inv }} \approx 0.15 \mathrm{a}$ and $\Delta \mathrm{G} / \mathrm{G} \leq 0.2$. Note also the marked difference in period between the two phenomena.

The strong MHD activity preceding the event and continuing throughout the disruption sequence allows the rigid toroidal rotation hypothesis to be used for generation of the pseudoviews necessary for the tomographic inversion. The circular harmonic technique of Cormack [20], suitably modified to suppress ringing artifacts, is used for the reconstructions. The technical details are discussed in the Appendix.

\section{MINOR DISRUPTIONS}

Density limit disruptions are often preceded by the locking of a rotating mode to the wall. Though no wall locking is observed during this discharge, the frequency of the MHD signals is found to decrease by about $20 \%$ during the lead-up to the emission crash (see Fig. 12(e)). The similarity of the wall time constant and the observed mode period $(\sim 50 \mu \mathrm{s})$ implies that the decreasing mode frequency during the pre-disruptive phase might be due to interaction between the magnetic island and the wall. The slowing down of the magnetic island has a destabilizing influence, in particular for modes with the rational magnetic surface close to the wall [12]. However, the small change in mode frequency suggests that one or more other mechanisms are driving the disruption. This is confirmed by reconstructions of the $1 / 1$ and $2 / 1$ components, which show negligible radial shear in the mode structure during the slow down phase.

Spatio-temporal contours of the SXR measurements are displayed in Fig. 3(a). Because of the sharp fall-off in the emission outside $r \sim 0.7 \mathrm{a}$, the odd and even parity components of the fundamental and second 


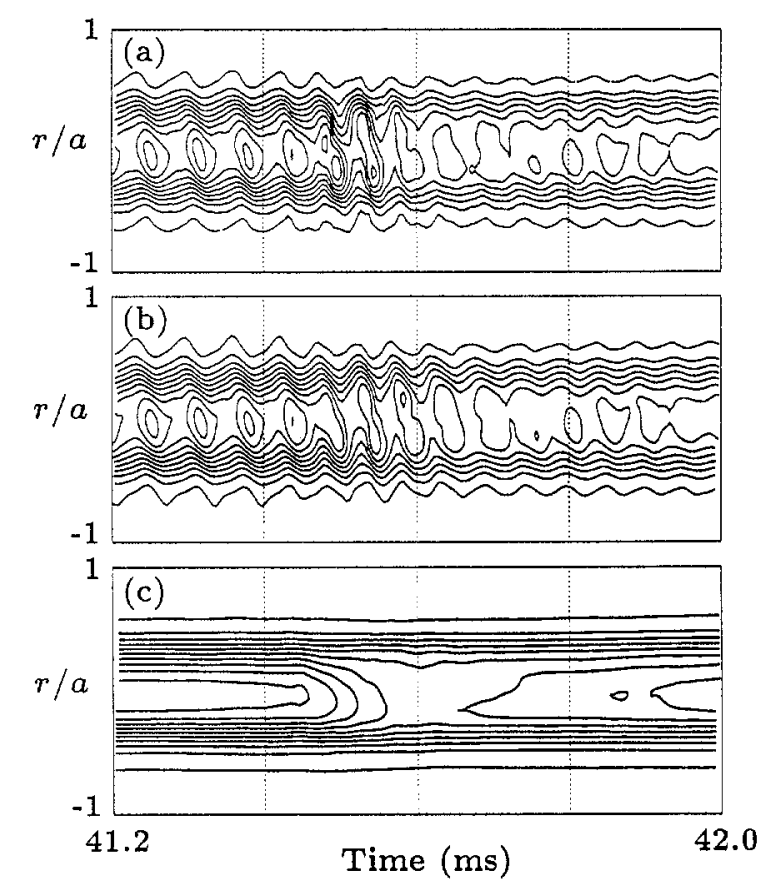

FIG. 3. Contours of SXR emission: (a) measured and (b) reconstructed; (c) background emission (oscillating component removed) obtained from (a). The contour levels in (a) and (b) are identical.

harmonic oscillations are attributed respectively to $1 / 1$ and $2 / 1$, and $3 / 2$ and $4 / 2$ MHD mode activity. The reliability of the $4 / 2$ reconstructions is somewhat uncertain owing to the likely coexistence of the identical parity and frequency $2 / 2$ mode. There is also some possible $5 / 2$ contamination of the $3 / 2$ mode. However, the second harmonic amplitude is an order of magnitude weaker than the fundamental, so that its influence on the reconstructed emission contours is not significant. The dominant helicities observed using toroidal and poloidal magnetic probes are $2 / 1$ and $3 / 1$. The mode assignments for the fundamental oscillation thus appear to be well justified, both a priori and, on the basis of the reconstruction, a posteriori.

The non-oscillatory (equilibrium) profile (Fig. 3(c)) evolves in a predominantly symmetric fashion (allowing simple Abel inversion). The centre of mass of the emission, however, exhibits a small amplitude $(\sim 1 \mathrm{~mm})$ oscillation synchronous with the cycle of disruptions. This small excursion is limited by the fast feedback position control system. The SXR geometry is appropriately corrected for this displacement at each processed time slice. The asymmetric component of the nonoscillatory emission must have toroidal mode number $\mathrm{n}=0$. Having odd parity, we ascribe its appearance to a possible $1 / 0$ component of the emission. In further support of this, we note that the m-th order projection component $\mathrm{g}_{\mathrm{m}}$ (p) (see Eq. (A.1)) must exhibit at least $(\mathrm{m} / 2)$ zeros in the range $(0,1)$ [20]. The absence of evidence for clear zeros in the odd parity component of the equilibrium contours is thus consistent with the $\mathrm{m}=1$ assignment. SXR measurements on JET using two arrays have also clearly established the existence of this $\mathrm{m}=1$ erosion [11]. Using a single view (at $\xi=90^{\circ}$, see Fig. 1), it is only possible to reconstruct the $\sin \theta$ component of the $1 / 0$ perturbation. This, however, at least allows us to monitor the temporal evolution of the $1 / 0$ mode amplitude, provided its orientation does not change with time.

Figure 4 shows a sequence of reconstructions (perspective plots and corresponding colour contours) through the disruption at $\mathrm{t} \sim 41.5 \mathrm{~ms}$. The images are rendered in the frame of reference rotating toroidally with the plasma. The velocity monitor is obtained from the central emission chord which is sensitive to the even perturbation components. As a check, the reconstructed wave form (Fig. 3(b)) obtained by projecting the (laboratory frame) tomographic images (excluding the $1 / 0$ component) shows excellent agreement with the experimental emission contours (Fig. 3(a)). Evident from Fig. 4 is the rapid emission decrease due either to an erosion of the emission profile or to the injection of a colder volume of plasma into the centre. The preand post-disruption SXR equilibrium profiles are symmetric (peaked and hollow, respectively) and so can be reconstructed without the rotation assumption. The tomographic images obtained under the rotation assumption satisfactorily account for the evolution of the SXR emission between these initial and final states. Computer animations show the post-disruption hollow emission profile to gradually 'fill in' and the emission increase in the Ohmic heating phase leading up to the next disruption.

\subsection{Mode energies}

The reconstructions are not inconsistent with penetration of a cold bubble to the centre as suggested in Ref. [14]. In these simulations the $2 / 1$ double tearing mode is destabilized by the hollow current profile created by the penetrating cold bubble. In an alternative scenario [13], a 'bubble-like' cooling of the plasma is the result of the initial destabilization of the $2 / 1$ mode and its subsequent non-linear coupling to other Fourier components. Unfortunately, the reconstructed images alone cannot distinguish the sequence of events. In an attempt to resolve this issue and to determine the role played by the different modes in the LT-4 disruptions, 


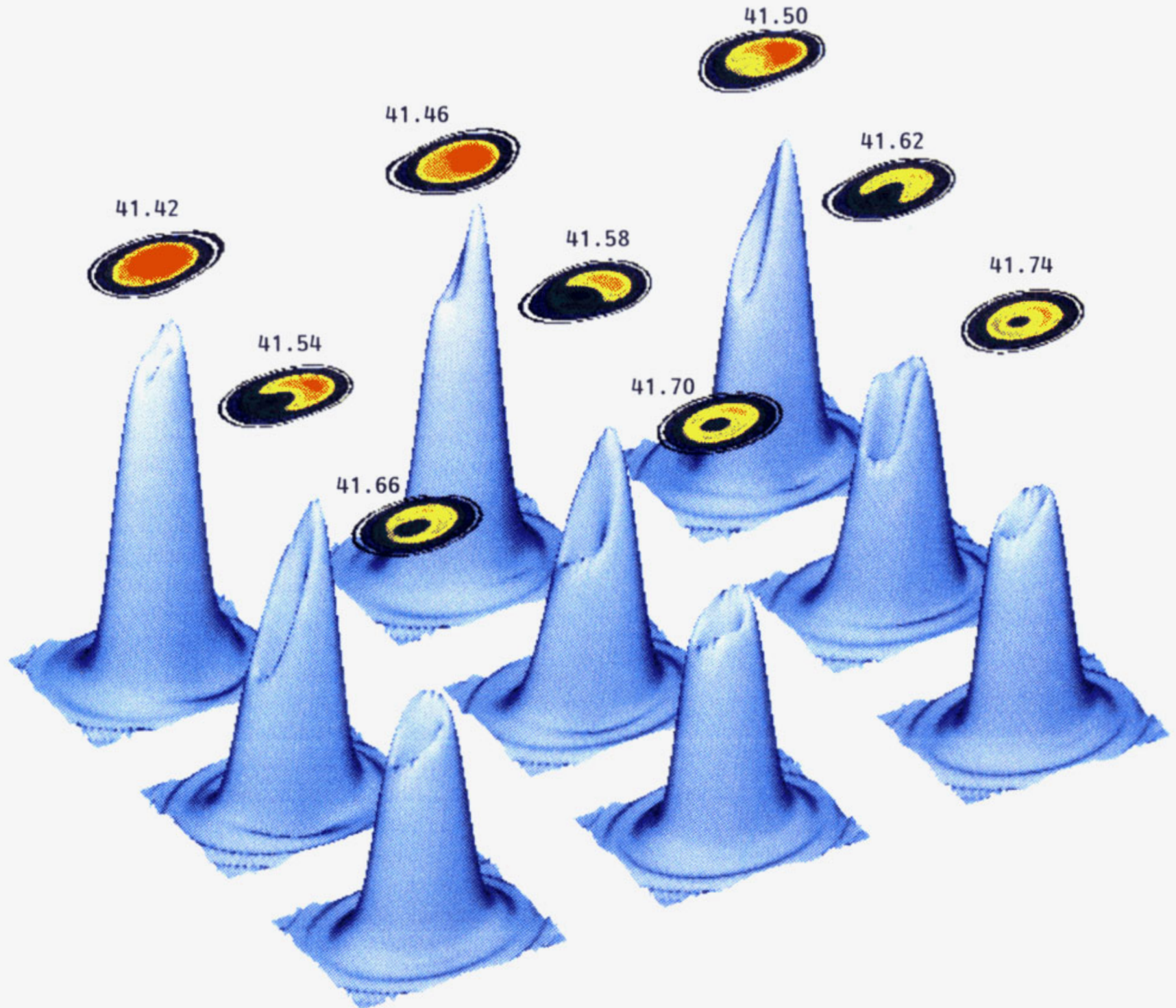

FIG. 4. Colour perspecive and elevated contour plots of the reconstructed emission contours during a minor disruption in LT-4. The snapshots are computed in the frame of reference rotating with the plasma and are shown at times equispaced by $20 \mu \mathrm{s}$ commencing at $t=41.43 \mathrm{~ms}$ and finishing at $t=41.73 \mathrm{~ms}$. Only the region between $-0.8 \mathrm{a}$ and $0.8 \mathrm{a}$ is shown.

we examine separately the spatio-temporal evolution of the various Fourier components.

Figure 5 shows the development of the spatial energy distribution (defined by Eq. (A.16)) of the equilibrium $(0 / 0$ and $1 / 0)$, fundamental $(1 / 1$ and $2 / 1)$ and odd parity second harmonic (3/2) components. Over time-scales longer than those shown in Fig. 5, a slow and pronounced outward shift in the maxima of the $1 / 1$ and $2 / 1$ emission contours leading up to the disruption is observed (see also Fig. 10). Since the total current remains relatively constant during this period (Fig. 2), this behaviour is consistent with a contracting current profile generally associated with density limit disruptions.

Integration of the spatial energy distribution over the plasma area gives the temporal behaviour of the total energy $\left\langle e_{m / n}, e_{m / n}\right\rangle_{w_{2}}$ (see Eq. (A.12)) of the 0/0, 1/1, $2 / 1,3 / 2$ and $1 / 0$ components of the emission, as displayed in Fig. 6. The oscillations in the $2 / 1$ energy at the time of the $0 / 0$ crash are artifacts of the signal fitting and reconstruction procedure, while the residual level of the 1/0 component between crashes is due to a small ( $\leqslant 0.1 \%$ ) systematic error in the location of the centre of mass of the $n=0$ emission. Also plotted are the energy histories for the most significant helical components $(m=2,3,4 / n=1)$ registered by the magnetic probe array. The second harmonic magnetic signals are smaller in amplitude by an order of magnitude and so are not considered further.

In Fig. 6 it can be seen that the onset of significant $1 / 1,1 / 0$ and $3 / 2$ activity occurs at the time when the 

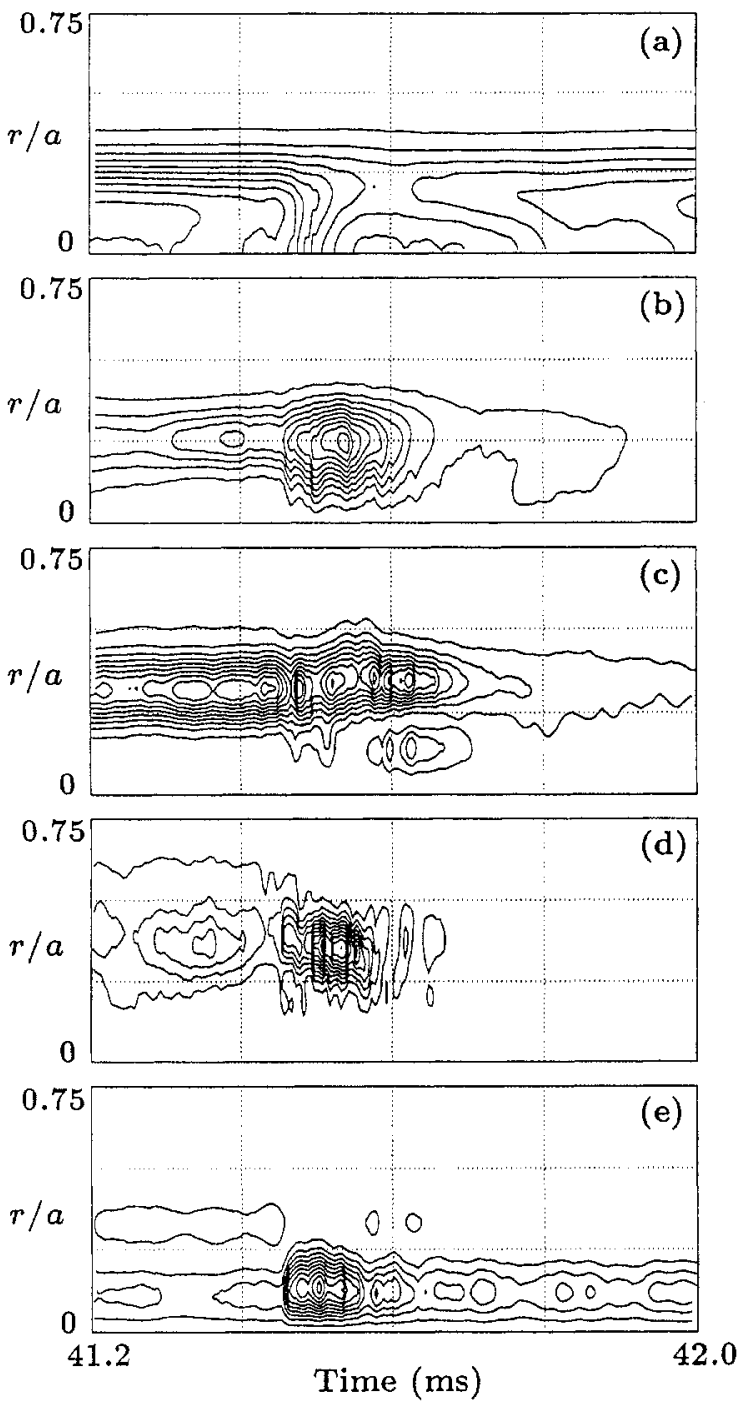

FIG. 5. Temporal evolution of the spatial distribution of the SXR emission in (a) the background, (b) the fundamental 1/1, (c) the fundamental $2 / 1,(d)$ the second harmonic $3 / 2$, and (e) the 110 component of the emission.

$2 / 1$ activity attains its maximum amplitude. Synchronous with this switch-on is the beginning of the $0 / 0$ crash. This suggests that the growth of the $1 / 1$ perturbation and the subsequent rapid cooling may be triggered by (or is at least coexistent with) the $2 / 1$ mode. Comparing Figs 5 and 6 , we further note that the radial distribution of the $2 / 1$ mode appears to bifurcate into two zones as the $1 / 1$ mode reaches its maximum and decays. The persistence of the feature and its reproducible appearance at each emission crash renders it unlikely to be a reconstruction artifact. Moreover, it is not an artifact caused by a sudden shift in the plasma axis during the crash: first, there is no evidence for such a shift in the equilibrium contours (Fig. 3(c)); second, introduction of an artificial axis shift during the time before the bifurcation fails to reproduce the observed behaviour. The splitting of the mode structure might suggest the appearance of a second $q=2$ surface and the associated double tearing mode as proposed in Ref. [14]. However, while in the simulations a strong double tearing mode reconnects all the flux inside the $\mathrm{q}=2$ surface, the experimentally observed $\mathrm{m}=2$ emission from the inner region is relatively weak. This, together with both the limited radial bandwidth of the image (see Fig. 13) and the strong apodictic effects of the centrally weighted emission, suggests that this interpretation should be viewed with caution. Nevertheless, a marked and consistent change in the $2 / 1$ structure appears to be precipitated by the bubble injection.

This temporal sequence of events, namely the excitation of a spectrum of modes by a high level of $2 / 1$ activity, seems to match well the numerical results of Ref. [13]. Evidence for mode coupling is found in the similar temporal behaviour of the $1 / 0,1 / 1$ and $3 / 2$ components. The considerable spatial and temporal overlap of the $1 / 0,1 / 1$ and $2 / 1$ components also supports the possibility of mode coupling, as suggested in Ref. [13]. However, while the $3 / 2$ mode plays a major role in the simulations, the corresponding SXR component is weaker by an order of magnitude than the $\mathrm{n}=1$ modes.

The magnetic activity mirrors the SXR behaviour, although throughout, and in agreement with the internal nature of the SXR mode, there is no detectable 1/1 perturbation at the plasma surface. We note the persistence of the $2 / 1$ activity in both the coil and the SXR signals even as the other modes decay. It is also interesting to observe that the $\mathrm{m}=4$ component appears to peak $\sim 50-100 \mu$ s after the peak of the $3 / 1$ and $2 / 1$ modes. Of course, the proximity of the mode also influences the coil signal and the deduced energy history. Nevertheless, with $\mathrm{q}_{\mathrm{a}} \leq 4$, and with the current and temperature profiles flattened and broadened by the earlier phase of the disruption, the appearance of an $\mathrm{m}=4$ kink mode in the final phase would seem to agree with simulations [13].

\subsection{Phase relationships}

It is important to examine the phase relationship between magnetic pick-up coil signals and the loworder angular modes visible in the emission. Assuming that the mode is moving with the plasma, the $\mathrm{m} / \mathrm{n}$ 

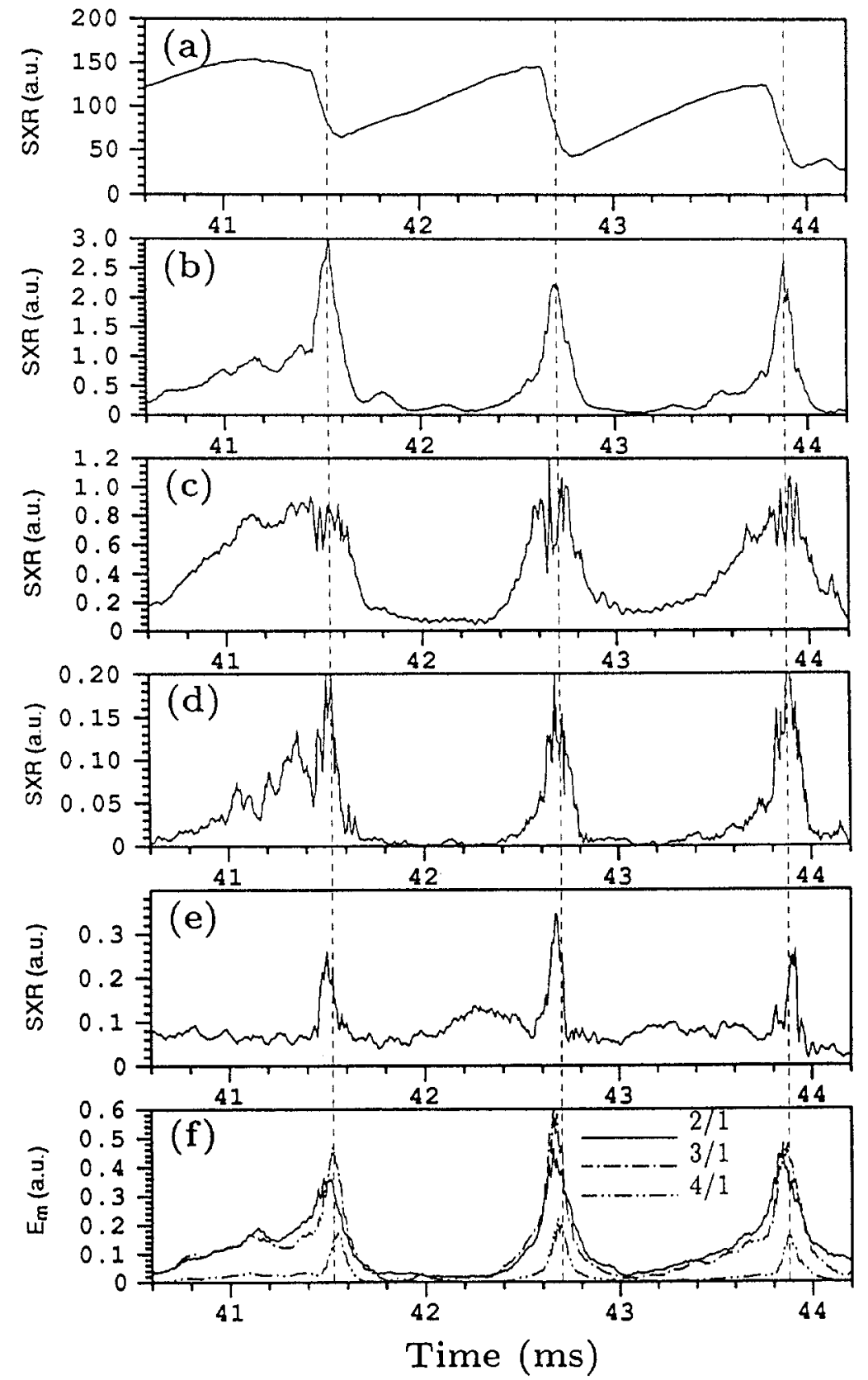

FIG. 6. Temporal evolution of the energy in the various Fourier components: (a) SXR background, (b) SXR odd parity fundamental $1 / 1$, (c) SXR even parity fundamental $2 / 1$, (d) $S X R$ odd parity second harmonic 3/2, (e) $S X R$ odd parity $1 / 0$, and $(f)$ magnetic activity in the various poloidal modes. The vertical scales are in arbitrary units; for (a) to (e) the relativity of the scales is maintained. 


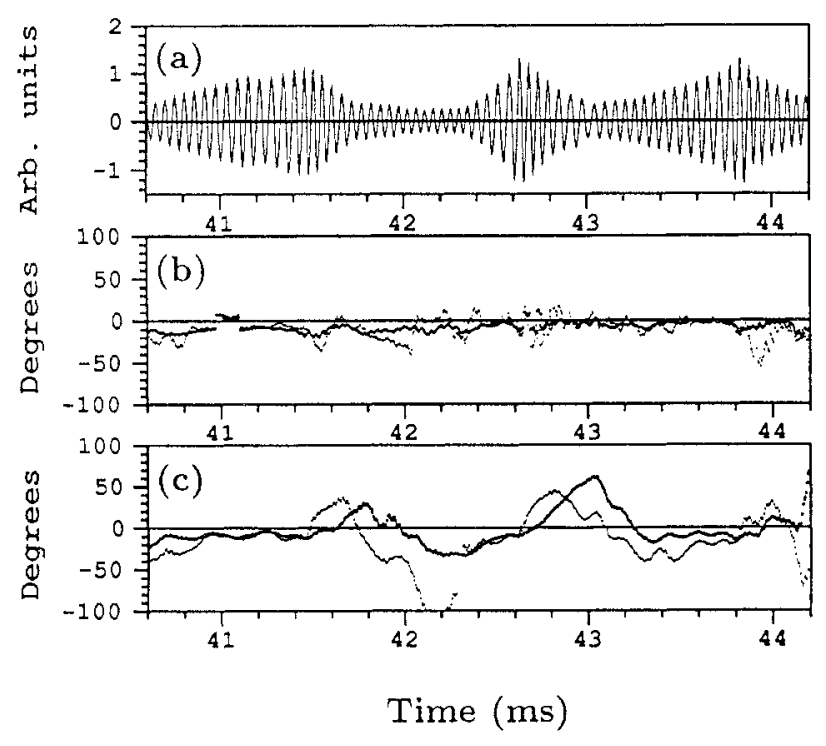

FIG. 7. (a) $2 / 1$ component of the magnetic perturbation at $\theta=0$ during part of the disruption sequence.

(b) Calculated phase difference $\epsilon_{2}$ between the reconstructions of the $2 / 1$ mode at radius $r=0.4 a$ and the $2 / 1$ magnetic field perturbation (bold dots) and $\epsilon_{2}$ for the $1 / 1$ mode at radius $r=0.3 a$ (faint dots). Note that the $2 / 1$ magnetic and SXR perturbations are in phase and locked throughout the disruption sequence; the 1/1 SXR component also appears to be phase locked to the $2 / 1$ activity.

(c) Phase of the 3/1 (bold dots) and 4/1 (faint dots) magnetic modes with respect to the $2 / 1$ magnetic activity. Note the phase shearing coincident with and following the disruption crashes.

magnetic perturbation at the coil radius and in the laboratory frame (with $\phi=0$ ) can be written

$\mathrm{B}_{\mathrm{m}}(\mathrm{t})=\mathrm{b}_{\mathrm{m}}(\mathrm{t}) \cos [\mathrm{m} \theta+\mathrm{n} \varphi(\mathrm{t})]$

where $b_{m}(t)$ is the instantaneous mode amplitude and

$\varphi(\mathrm{t})=\int \Omega(\mathrm{t}) \mathrm{dt} \quad \Omega(\mathrm{t})=\mathrm{v}_{\mathrm{T}}(\mathrm{t}) / \mathrm{R}$

where $v_{T}(t)$ is the instantaneous toroidal angular velocity. The structure of the m-th order poloidal SXR component at the same toroidal location can be expressed in terms of the experimentally determined coefficients $q_{m \nu}(t)$ (see Eq. (A.1)) as

$$
\begin{aligned}
& \mathrm{E}_{\mathrm{m}}(\mathrm{r}, \theta, \mathrm{t})=\exp (\operatorname{im} \theta) \sum_{\nu=\mathrm{m}}^{\nu \max } \mathrm{q}_{\mathrm{m} \nu}(\mathrm{t}) \mathrm{R}_{\mathrm{m} \nu}(\mathrm{r})+\mathrm{c} . \mathrm{c} \\
& \quad=A_{\mathrm{m}}(\mathrm{r}, \mathrm{t}) \cos \left[\mathrm{m} \theta+\eta_{\mathrm{m}}(\mathrm{r}, \mathrm{t})\right]
\end{aligned}
$$

Allowing for the toroidal rotation, we can write

$\eta_{\mathrm{m}}(\mathrm{r}, \mathrm{t})=\mathrm{n} \varphi(\mathrm{t})+\mathrm{m} \epsilon_{\mathrm{m}}(\mathrm{r}, \mathrm{t})$

where the residual phase $\epsilon_{\mathrm{m}}(\mathrm{r}, \mathrm{t})$ represents the possibly time varying radial shear of the mode and also measures the relative phase between the emission and magnetic perturbations associated with the islands.

It is found that over regions of significant emission (within the contours of Fig. 5) the maximum spatial variation of the phases $\epsilon_{2}$ and $\epsilon_{1}$ amounts to $\leqslant 30^{\circ}$. The absolute phase of the SXR components $E_{1}$ (at $r=0.3$ ) and $E_{2}$ (at $r=0.4$ ) with respect to the $m=2$ component of the coil array signals (at $\theta=0$ ) is shown in Fig. 7. The $1 / 1$ and $2 / 1$ oscillations are strongly coupled (phase locked) throughout the mode activity. Moreover, the $2 / 1$ magnetic and emission perturbations are in phase $\left(\epsilon_{2} \approx 0\right)$, as expected for modes of this nature. The phases $\epsilon_{1}$ and $\epsilon_{2}$ thus appear to be both space and time invariant. These results validate the invoked assumption of rigid body rotation and are also consistent with the results of Ref. [13] where modes with different helicity $\mathrm{m} / \mathrm{n}$ are phase locked during times of high activity, with the $\mathrm{O}$-point of the magnetic islands overlapping at a fixed toroidal position. Since a strong interaction between the magnetic islands and the wall should lead to a radially sheared phase of the mode [12], the experimental results indicate that the modewall interaction plays only a small role for these interior modes during minor disruptions in LT-4.

Though the $1 / 1$ and $2 / 1$ SXR modes appear always to be phase locked, the magnetic coils show some phase shear $\left(\Sigma 90^{\circ}\right)$ between the higher $m$ components during and immediately following the minor disruptions (mainly when the mode amplitudes are small - see Fig. 7). The components appear to synchronize during the growth phase, in agreement with simulation [13]. During the quench phase of the major disruption, however, the $3 / 1$ and $4 / 1$ modes shift by $\sim 180^{\circ}$ with respect to the $2 / 1$ component in a single oscillation cycle. This is discussed further in the next section.

\section{THE MAJOR DISRUPTION}

We now analyse the terminating disruption at $t \approx 47 \mathrm{~ms}$ and address the question of what it is that makes this disruption a major one. Snapshots of the reconstructed SXR emission during this event are shown in Fig. 8. Once again, the agreement between the measured and reconstructed line integral signals is found to be satisfactory (Fig. 9). As for Fig. 4, the 


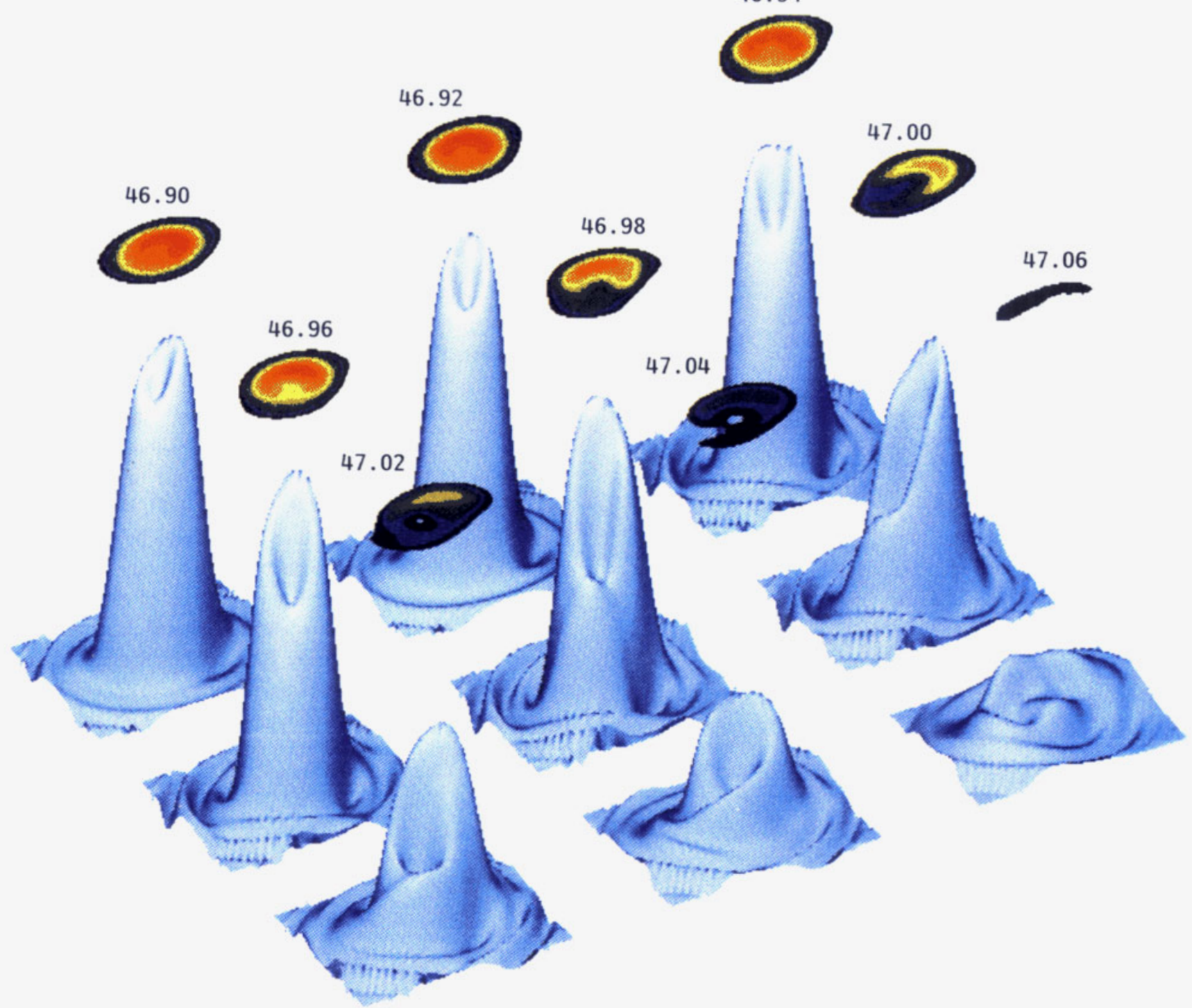

FIG. 8. Colour perspective and elevated contour plots of the reconstructed emission contours during a major disruption in LT-4. The snapshots are computed in the frame of reference rotating with the plasma and are shown at times equispaced by $20 \mu s$ commencing at $t=46.90 \mathrm{~ms}$ and finishing at $t=47.06 \mathrm{~ms}$. Only the region between $-0.8 a$ and $0.8 a$ is shown.

images are translated to the plasma frame. The emission levels in Fig. 8 have been scaled by a factor of 1.5 compared with the reconstructions of Fig. 4. In comparing the reconstructions, we first note the similarity of the relative phases of the emission erosion consistent with the already noted fixed relative phase between the $1 / 1$ and 2/1 components. As for the minor disruptions (Fig. 4), a 'bubble-like' erosion associated with the $1 / 1$ perturbation appears to inject colder edge plasma into the centre of the discharge. However, in constrast to the minor disruption, the emission appears to quench before the penetration is complete. The $\mathrm{m}=0$ energy distribution contours at and immediately following the emission quench at $t \approx 47.05 \mathrm{~ms}$ show a flattening of the emission profile consistent with destruction of the magnetic surfaces and field line stochastization.

The temporal evolution of the spatial distribution of the background, 1/1 and 2/1 SXR emission components is shown in Fig. 10. Again, there is a strong similarity to the minor disruptions shown in Fig. 5. The same hollow background profile is created and the weak bifurcation in the $2 / 1$ signal is evident. The temporal evolution of the SXR energy in the $1 / 1$ and $2 / 1$ Fourier components is shown in Fig. 11(a) for the last minor disruption and the major disruption. The magnetic activity of the $2 / 1,3 / 1$ and $4 / 1$ modes as determined from the edge pick-up coils is shown in Fig. 11(b). Though the coil set cannot reliably represent the low 

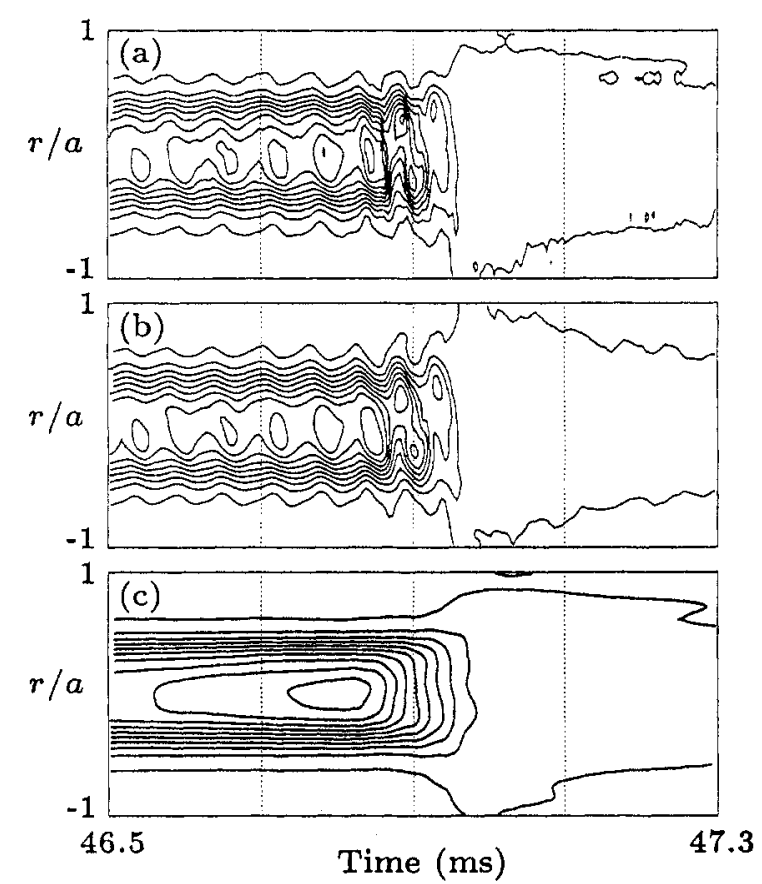

FIG. 9. Contours of SXR emission: (a) measured and (b) reconstructed; (c) background emission (oscillating component removed) obtained from (a). The contour levels in (a) and (b) are identical.

order modes $m \leq 4$ in the case of broadband activity, we assume for simplicity that the aliasing contamination is small. Note the similarity in the behaviour of the $1 / 1$ and $2 / 1$ SXR modes between the penultimate disruption and the final disruption. When normalized to the total emission, however, it is found that the energy of the $n=1$ components during the major disruption is greater by a factor of two than that during the minor disruptions. Another significant difference is the dramatic increase in the $3 / 1$ and $4 / 1$ magnetic activity occurring after the SXR emission peak at $47.0 \mathrm{~ms}$. Associated with this increased activity is a sudden (within $100 \mu \mathrm{s}$ ) shifting in phase of $\sim 180^{\circ}$ between the $3 / 1$ and $4 / 1$ magnetic components relative to the $2 / 1$ mode (Fig. 11(c)). Though the minor distuptions excite a shearing of phase of the external $3 / 1$ and $4 / 1$ modes, this is repaired during the growth phase leading to the next disruption. This is not so for the major disruption, suggesting that the flat profile attending the stochastization of the magnetic surfaces destabilizes the $3 / 1$ and $4 / 1$ external kink modes, ultimately destroying the magnetic configuration. Note that this occurs after the collapse of the SXR emission and so does not affect the validity of the rotation assumption.

\section{SUMMARY}

Tomographic reconstruction of the SXR signals during density limit disruptions in the LT-4 tokamak suggests a penetration to the centre of an outer colder plasma region. The energy history of the emission components indicates that the growth of the $1 / 1$ and $3 / 2$ modes is triggered by a pre-existing high level of $2 / 1$ activity. The subsequent bifurcation of the $2 / 1$ spatial distribution appears to be initiated by the creation of a hollow emission profile attending the bubble injection and may be associated with the formation of a $2 / 1$ double tearing mode. The phase distributions for the $n=1$ SXR components show little radial variation, while the $1 / 1$ and $2 / 1$ components are found to be locked in phase with the $2 / 1$ magnetic perturbation. In addition, the 2/1 magnetic and SXR X-points are found to coincide throughout the disruption sequence. These results support the invoked assumption of rigid body rotation.

The observed cold bubbles are similar to those observed during sawtooth crashes [21] and disruptions [11] in JET. The penetrating cold bubble and bifurcation of the $2 / 1$ mode are in agreement with recent numerical work [14]. However, at odds with this model, a high level of $2 / 1$ activity seems to accompany,
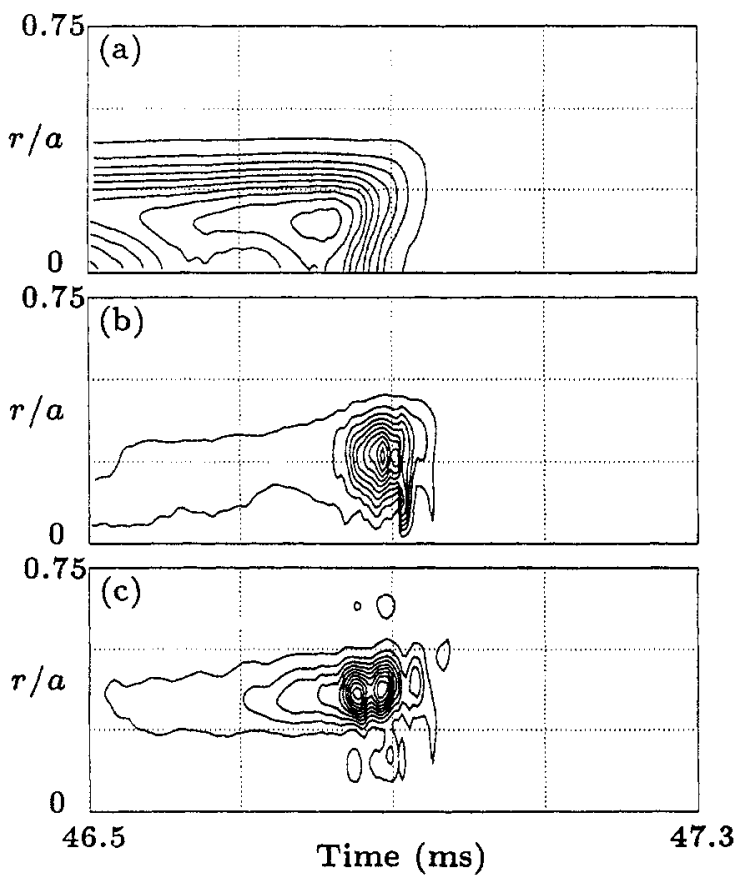

FIG. 10. Temporal evolution of the spatial distribution of the SXR emission in (a) the background, (b) the fundamental 1/1, and (c) the fundamental 2/1 components during the final disruptive phase. 

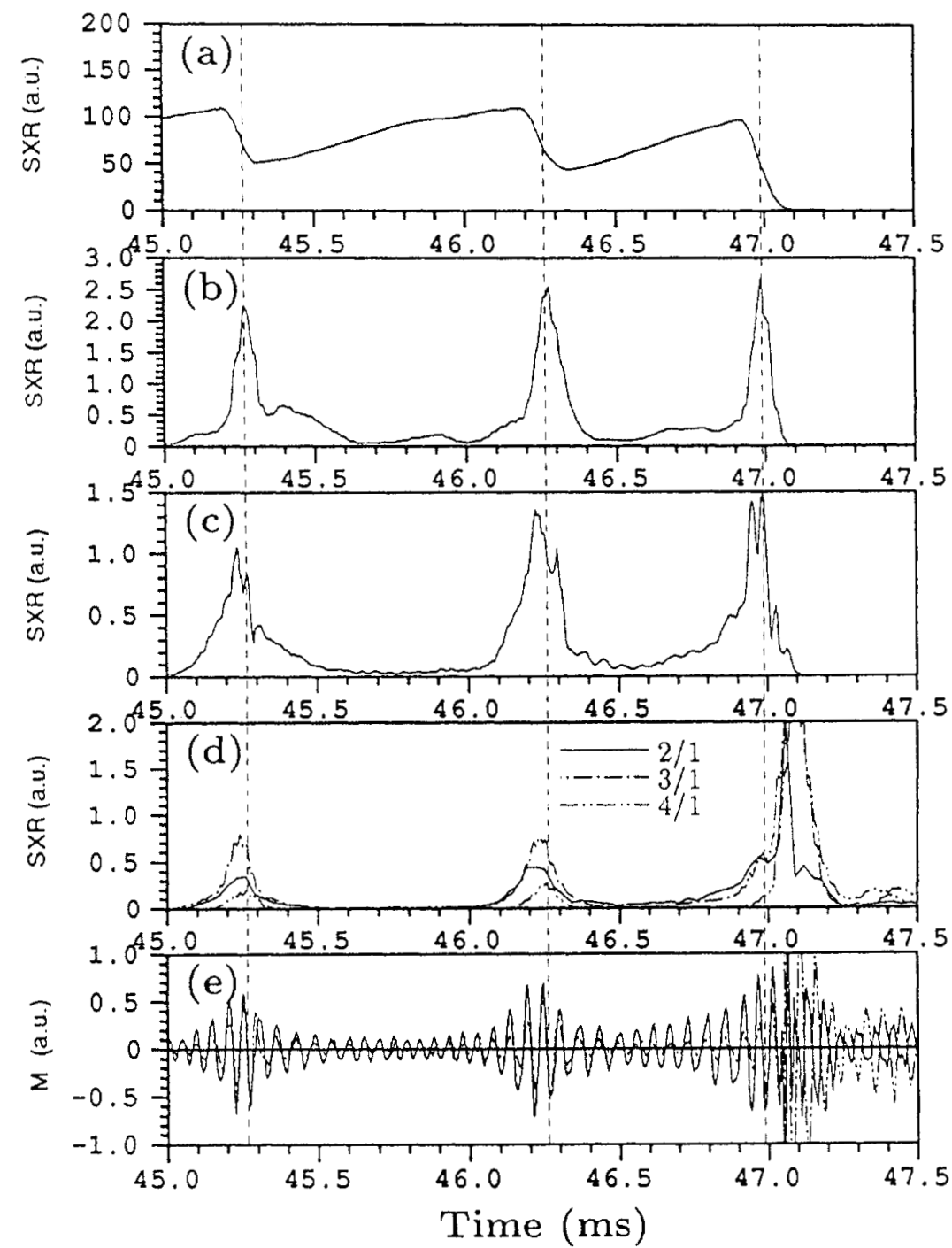

FIG. 11. Temporal evolution of the energy in the various Fourier components: (a) SXR background, (b) SXR odd parity fundamental $1 / 1$, (c) SXR even parity fundamental $2 / 1,(d)$ magnetic activity in the various poloidal modes (note the strong $3 / 1$ and 4/1 activity following the final crash), and (e) magnetic signals for the $2 / 1$ (solid) and $4 / 1$ (broken) components showing the $180^{\circ}$ phase shift after the final disruption.

if not directly stimulate, the $1 / 1$ bubble injection. The temporal evolution of the various SXR mode energies appears to be in good agreement with the results of Ref. [13]. The observation of a $1 / 0$ mode, indicating possible mode coupling effects, the observed fixed phase relation between the $n=1$ SXR components, and the growth of an $\mathrm{m}=4$ mode during the final phase of the disruption are all in qualitative agreement with Ref. [13]. At odds with Ref. [13] is the persistent small $3 / 2$ activity throughout the disruption events. This is also in contrast to observations in JIPP T-II [22], where the $3 / 2$ activity appears to be important.
The initial phase of the major disruption, with a rapid growth of the $1 / 1$ mode from a pre-existing high level of $2 / 1$ activity, is similar to the minor disruptions. However, during the phase leading to the major crash, the normalized (with respect to the background emission) mode energies are greater by a factor of around two. The emission collapse of the major disruption is also followed by a dramatic increase in the level of the magnetic activity. This is consistent with observations in JET [4] and with numerical simulations [13]. It also fits well the scenario where stochastic field lines created by the interacting magnetic islands during the disruption 
broaden the current profile and degrade the configuration [23]. If the magnetic surfaces heal, the plasma survives and the disruption is minor. However, the current profile, broadened by the recurring minor disruptions, will eventually become kink unstable. The appearance of the strong magnetic activity during the final emission collapse is suggestive of the latter.

Finally, the extent to which the appearance of colder regions is due to limitations of the reconstruction process has recently attracted considerable attention [24-26]. These studies have focused on rotation free reconstruction issues and therefore do not directly bear on the techniques used in this work. However, our reconstructions suggest, in agreement with recent simulations [24], that the bubbles are not merely reconstruction artifacts. The theoretical understanding of the cold bubbles is relatively poor. While a few different models have been proposed for the bubble injection during sawtoothing [27, 28], external kink modes [29] and disruptions [14], further work, both theoretical and experimental, is needed to determine their true nature.

\section{Appendix}

\section{TOMOGRAPHIC TECHNIQUES}

\section{Rigid rotation}

Time-frequency analysis indicates that the SXR signals during the disruption sequence can be modelled as the sum of a DC component together with the fundamental oscillation and its second harmonic. Since there is no measurable dispersion in the MHD mode frequency across the plasma section (this is confirmed by the reconstructions) the assumption of rigid toroidal rotation is invoked to allow inversion of the oscillating amplitudes [18]. The identities of the associated helical modes are obtained from the parity (poloidal mode number $\mathrm{m}$ ) and frequency (toroidal mode number $n$ ) properties of the oscillations. The validity of the assumption of rigid rotation has been recently verified (albeit during relatively quiescent activity) using three poloidally separated detector arrays [24].

During LT -4 disruptions, the mode oscillation period is not significantly less than the duration of the emission crash, so that application of the rigid rotation technique is not straightforward. Fourier methods are unsuitable for extraction of the oscillation phase and amplitude and of the instantaneous equilibrium emission necessary for the tomographic inversion. To overcome this problem, we assume that the DC, fundamental and second harmonic components dominate the emission behaviour throughout the crash phase. The free model parameters (amplitude, frequency and phase) are fitted using a constrained non-linear least squares algorithm in a fixed-width time window that moves incrementally along the digitized signal wave form. The parameters are time independent within this window (as required by the rigid rotation hypothesis), but are independently determined at each time step and for each signal channel. This accommodates the observed time varying oscillation frequency (given appropriate choice of window width and step size) and allows for phase shear between signals at different impact parameters. The width of the fitting window is typically comparable to the oscillation period, so that features changing dramatically within this time interval will be smoothed. Nevertheless, the algorithm has successfully decomposed synthetic wave forms similar to the measured signals into their component parts (see Fig. 12), giving confidence that the properties of the oscillating components of the emission throughout the relatively rapid crash phase can be successfully characterized.

\section{Tomography algorithm}

We utilize the orthogonal expansion technique [20] for reconstruction of emissivity contours. As will be seen, the method can be adapted to incorporate a priori constraints. In particular, we suppress edge ringing artifacts due to noise on the signals by constraining the energy of the image in regions of low emission. This approach can also be applied in cases where the viewing access is partly occluded or the views are incomplete.

First, we state the basic important relationships between the emissivity $E$ (confined to the unit disc $D$ ) and its projections. Let $\mathrm{G}(\mathrm{p}, \xi)$ be the line integral (radon transform) of $E(r, \theta)$ along the straight line $r \cos (\theta-\xi)=p$ and write this mapping as $G=P E$. The geometry is illustrated in Fig. 1. Both $E$ and $G$, being confined to $\mathrm{D}$, can be expanded as a Fourier series

$$
\mathrm{E}(\mathrm{r}, \theta)=\sum_{\mathrm{m}} \exp (\operatorname{im} \theta) \mathrm{e}_{\mathrm{m}}(\mathrm{r})
$$

$$
\mathrm{G}(\mathrm{p}, \xi)=\sum_{\mathrm{m}} \exp (\mathrm{im} \xi) \mathrm{g}_{\mathrm{m}}(\mathrm{p})
$$



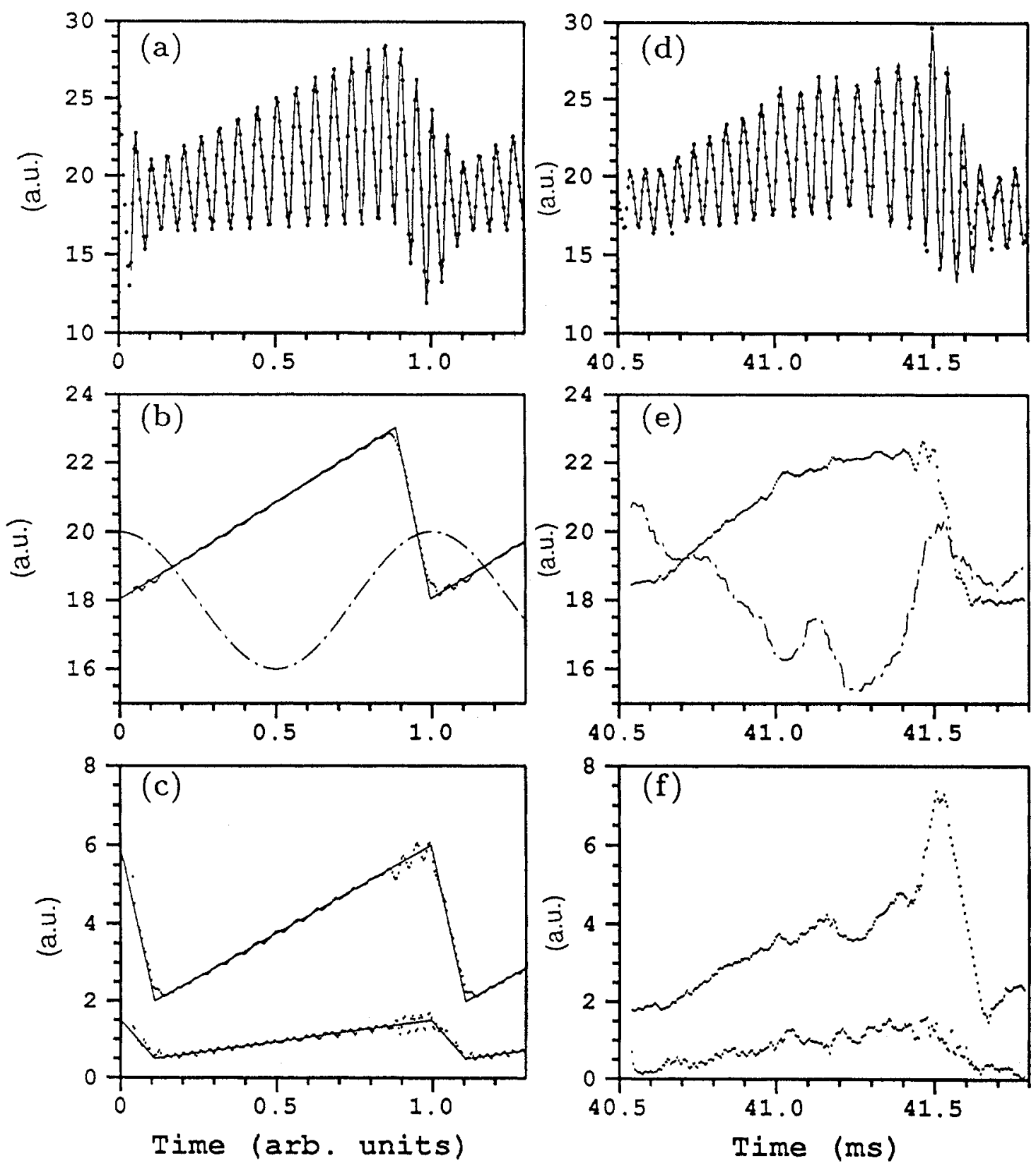

FIG. 12. Left-hand side: decomposition of synthetic test function, right-hand side: decomposition of the typical experimental signatures into their component parts.

(a) and (d) Input signals; the raw signal is represented by dots and the signal reconstructed from its component parts is represented by the solid line. (b) and (e) Best fit DC component (dotted line, arb. units) and instantaneous frequency (broken line, $\mathrm{kHz}$ units). (c) and (f) Fundamental and second harmonic component amplitudes. The solid lines in (b) and (c) are the input test function parameters. 
with components $e_{m}$ and $g_{m}$ (the toroidal subscript $n$ is here understood). Cormack [20] identified the following, particularly useful, representation for $\mathrm{P}$ :

$\mathrm{P}\left[\mathrm{R}_{\mathrm{m} \nu}(\mathrm{r}) \exp (\operatorname{im} \theta)\right]=\sigma_{\nu} \mathrm{V}_{\nu}(\mathrm{p}) \exp (\mathrm{im} \xi)$

where $\mathrm{R}_{\mathrm{m} \nu}(\mathrm{r})$ are the Zernike circle polynomials and the $V_{\nu}(p)$ are given by

$\mathrm{V}_{\nu}(\mathrm{p})=\frac{2}{\sqrt{\pi}}\left(1-\mathrm{t}^{2}\right)^{1 / 2} \mathrm{U}_{\nu}(\mathrm{p})$

where the $U_{\nu}(p)$ are Chebyshev polynomials of the second kind. The radial index $\nu$ takes allowable values $\nu=|\ell|+2 \mathrm{~s}$ for $\mathrm{s}=0,1,2, \ldots$, and the scalars are

$\sigma_{\nu}=\left[\frac{2 \pi}{\nu+1}\right]^{1 / 2}$
For convenience, we denote the disc and projection space functions as $u_{m \nu}(r, \theta)=R_{m \nu}(r) \exp (\operatorname{im} \theta)$ and $\mathrm{v}_{\mathrm{m} \nu}(\mathrm{p}, \xi)=\mathrm{V}_{\nu}(\mathrm{p}) \exp (\mathrm{im} \xi)$, respectively. The set $\left\{\mathrm{u}_{\mathrm{m} \nu}\right\}$ forms an orthonormal basis for the Hilbert space constructed on $\mathrm{D}$ with uniform weight $\mathrm{w}_{2}=1$ and inner product

$\left\langle E_{1}, E_{2}\right\rangle_{w_{2}}=\int_{0}^{2 \pi} \frac{d \theta}{2 \pi} \int_{0}^{1} r d r w_{2} E_{1} E_{2}^{*}$

Similarly, the set $\left\{v_{m \nu}\right\}$ is an orthonormal basis spanning the projection space PD with inner product

$\left\langle\mathrm{G}_{1}, \mathrm{G}_{2}\right\rangle_{\mathrm{w}_{1}}=\int_{0}^{2 \pi} \frac{\mathrm{d} \xi}{2 \pi} \int_{-1}^{1} \mathrm{dtw}_{1} \mathrm{G}_{1} \mathrm{G}_{2}^{*}$

and weight $\mathrm{w}_{1}=\left(2 \sqrt{1-\mathrm{t}^{2}}\right)^{-1}$. With these definitions, $G$ and $E$ can be expanded as
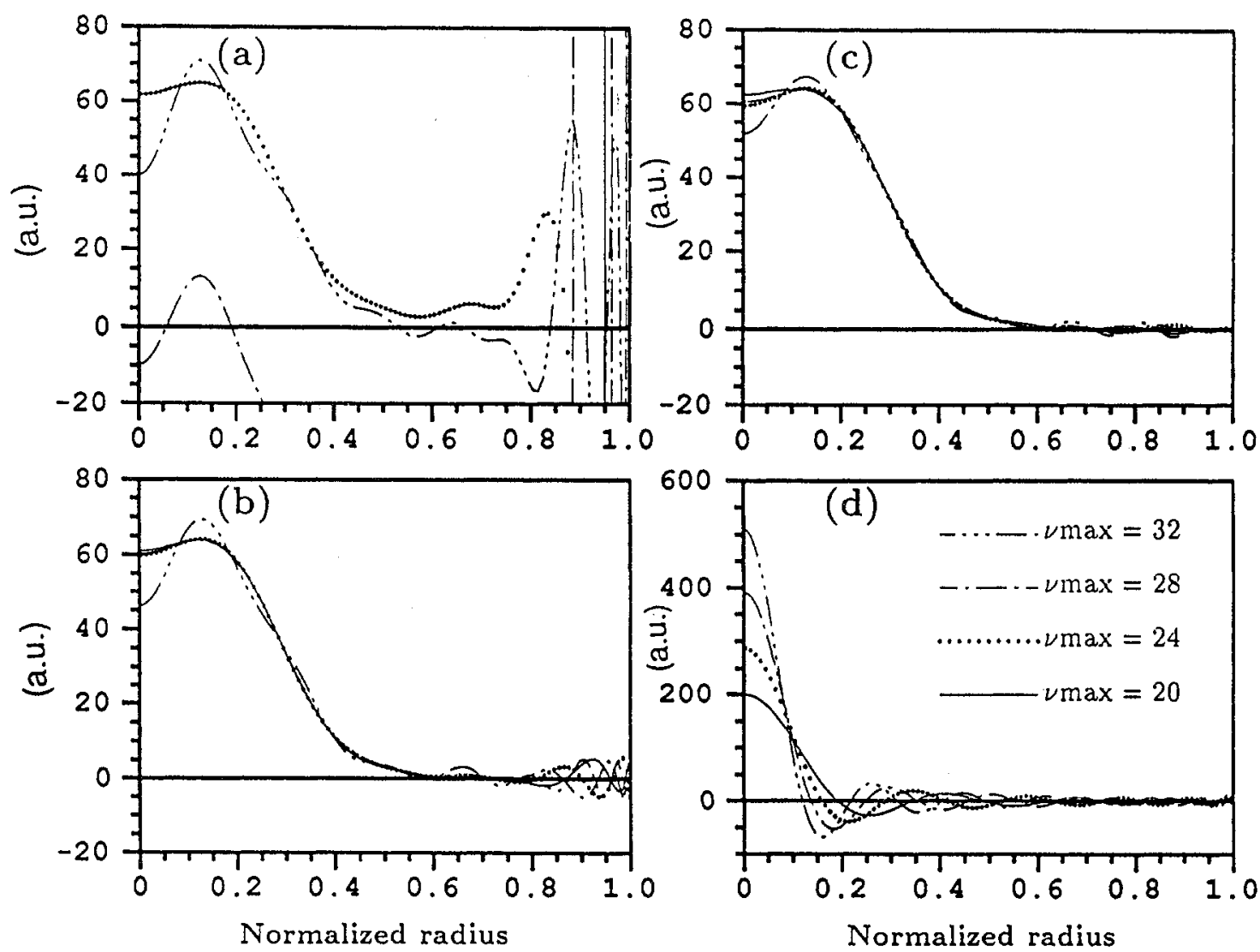

FIG. 13. (a) Unconstrained least squares fits to the circularly symmetric (equilibrium) part of the SXR emission profile at $t=41.0 \mathrm{~ms}$. The four curves correspond to maximum values of the radial expansion order, $\nu_{\max }=20$, 24, 28, 32. Note the strong sensitivity of the reconstructions to the small noise component of the signal.

(b) and (c) Corresponding energy constrained reconstructions for $\zeta_{p}(0.9,1.0)=0.01$ and 0.0001 , respectively. (d) Reconstructions of a point source located at the origin, showing the improvement in resolution obtained with increasing radial expansion order. 
$\mathrm{E}(\mathrm{r}, \theta)=\sum_{\mathrm{m}=-\infty}^{\infty} \sum_{\nu=\mathrm{m}}^{\infty} \mathrm{q}_{\mathrm{m} \nu} \mathrm{u}_{\mathrm{m} \nu}(\mathrm{r}, \theta)$

$\mathrm{G}(\mathrm{p}, \xi)=\sum_{\mathrm{m}=-\infty}^{\infty} \sum_{\nu=\mathrm{m}}^{\infty} \mathrm{p}_{\mathrm{m} \nu} \mathrm{v}_{\mathrm{m} \nu}(\mathrm{p}, \xi)$

where the complex Fourier coefficients, or moments of $\mathrm{G}$ and $\mathrm{E}$, are given by the inner products

$\mathrm{p}_{\mathrm{m} \nu}=\left\langle\mathrm{G}, \mathrm{v}_{\mathrm{m} \nu}\right\rangle_{\mathrm{w}_{1}}$

$\mathrm{q}_{\mathrm{m} \nu}=\left\langle\mathrm{E}, \mathrm{u}_{\mathrm{m} \nu}\right\rangle_{\mathrm{w}_{2}}$

These representations for $\mathrm{G}$ and $\mathrm{E}$ give the singular value decomposition of the projection operator $\mathrm{P}$ and allow the reconstructed image to be expressed in terms of the projection moments $\mathrm{p}_{\mathrm{m} \nu}$ :

$$
\begin{aligned}
\mathrm{E} & =\mathrm{P}^{-1} \mathrm{G} \\
& =\sum_{\mathrm{m} \nu} \frac{\mathrm{p}_{\mathrm{m} \nu}}{\sigma_{\nu}} \mathrm{u}_{\mathrm{m} \nu}
\end{aligned}
$$

The coefficients $\mathrm{q}_{\mathrm{m} \nu}=\mathrm{p}_{\mathrm{m} \nu} / \sigma_{\nu}$ are retrieved with mild ill-condition from $\mathrm{G}$, since the singular values $\sigma_{\nu}$ decrease slowly to zero with increasing radial order $\nu$. For $M$ complete views, however, only $\mathbf{M}^{2}+M$ of the recovered moments are free of angular aliasing contamination [30]. When the $M$ views are discretely sampled (incomplete), the retrieval of $\mathrm{E}$ becomes significantly more noise prone and susceptible to aliasing corruption. The latter problem can be eliminated when the views are effectively band limited and Nyquist sampled, as is the case for the LT-4 SXR camera. (Of course, the Cormack representation for $\mathrm{E}$, being spatially bounded, is then no longer strictly valid.)

For circular harmonic reconstruction techniques, the condition of the system design matrix (relating the discretized source to the line integral measurements) is optimum (unity) when $\mathrm{N}$ parallel chords are arranged to $t_{i}=\sin \left(\alpha_{i}\right)$, where the angles $\alpha_{i}$ are equispaced on $[0, \pi / 2]$. In such circumstances, the expansion coefficients $\mathrm{p}_{\mathrm{m} \nu}$ can be recovered by harmonic analysis over angles $\xi$ and $\alpha$. For equispaced chords, however, the condition number rapidly increases as the number of retrieved coefficients increases towards the maximum $\mathrm{N}$. The result is that the unconstrained fit oscillates wildly if the number of recovered coefficients is too great (see Fig. 13(a)) or if the data are noisy. Suppression of the ringing artifacts can be obtained at the expense of bandwidth by attempting to retrieve fewer Fourier coefficients from the available data.
An estimate of the reconstruction bandwidth is provided by the system point response $h\left(r, \theta ; r_{0}, \theta_{0}\right)$. This is given by the projection onto the image space $S$ (spanned by the finite $M$-view set $\left\{S_{M}\right\}$ of recovered basis functions $\mathrm{u}_{\mathrm{m} \nu}$ ) of the delta function $\Delta=2 \pi \delta\left(\theta-\theta_{0}\right) \delta\left(\mathrm{r}-\mathrm{r}_{0}\right) / \mathrm{r}[30]:$

$$
\begin{gathered}
\mathrm{h}_{s}\left(\mathrm{r}, \theta ; \mathrm{r}_{0}, \theta_{0}\right)=\sum_{\left\{\mathrm{s}_{\mathrm{M}}\right\}}\left\langle\Delta, \mathrm{u}_{\mathrm{m} \nu}\right\rangle_{\mathrm{w}_{2}} \mathrm{u}_{\mathrm{m} \nu}(\mathrm{r}, \theta) \\
\quad=\sum_{\left\{\mathrm{s}_{\mathrm{M}}\right\}} \mathrm{u}_{\mathrm{m} \nu}^{*}\left(\mathrm{r}_{0}, \theta_{0}\right) \mathrm{u}_{\mathrm{m} \nu}(\mathrm{r}, \theta)
\end{gathered}
$$

The reconstruction of an arbitrary emissivity profile $\mathrm{E}$ in the subspace $S$ is then given by

$\mathrm{E}_{S}\left(\mathrm{r}_{0}, \theta_{0}\right)=\sum_{\left\{\mathrm{s}_{\mathrm{M}}\right\}}\left\langle\mathrm{E}, \mathrm{u}_{\mathrm{m} \nu}\right\rangle \mathrm{u}_{\mathrm{m} \nu}\left(\mathrm{r}_{0}, \theta_{0}\right)=\left\langle\mathrm{E}, \mathrm{h}_{\mathrm{S}}\right\rangle_{\mathrm{w}_{2}}$

so that $h_{S}$ is the blurring to $\mathrm{E}$ due to the system resolution. The point response at the origin $\left(\mathrm{r}_{0}=0\right)$ for various radial expansion orders is shown in Fig. 13(d).

With the use of a priori knowledge it is possible to reduce noise sensitivity without compromising the reconstruction bandwidth. Since the available line integrals do not couple well, or at all, to outer regions of the object, and are affected by noise, knowledge of the likely object behaviour in that region, for example, can be used to stabilize the inversion. We choose to constrain the relative energy content of the reconstructed image in the unknown or poorly sampled region. Alternatively, we could elect to limit the energy content of the least squares fit to poorly sampled portions of the projections. The fractional energy enclosed between radii $r_{i}$ and $r_{0}$ for the $m$-th Fourier component is given by

$\zeta_{\mathrm{m}}\left(\mathrm{r}_{\mathrm{i}}, \mathrm{r}_{0}\right)=\left(\int_{\mathrm{r}_{\mathrm{i}}}^{\mathrm{r}_{0}} \mathrm{rdr}\left|\mathrm{e}_{\mathrm{m}}\right|^{2}\right) /\left\langle\mathbf{e}_{\mathrm{m}}, \mathrm{e}_{\mathrm{m}}\right\rangle_{\mathrm{w}_{2}}$

or, more explicitly,

$$
\begin{array}{r}
\zeta_{\mathrm{m}}\left(\mathrm{r}_{\mathrm{i}}, \mathrm{r}_{0}\right)=\frac{\sum_{\nu}\left(\mathrm{a}_{\mathrm{m} \nu}^{2}+\mathrm{b}_{\mathrm{m} \nu}^{2}\right) \mathrm{S}_{\nu \nu}^{\mathrm{m}}\left(\mathrm{r}_{\mathrm{i}}, \mathrm{r}_{0}\right)}{\sum_{\nu}\left(\mathrm{a}_{\mathrm{m} \nu}^{2}+\mathrm{b}_{\mathrm{m} \nu}^{2}\right)} \\
+\frac{2 \sum_{\nu} \sum_{\nu^{\prime}>\nu}\left(\mathrm{a}_{\mathrm{m} \nu} \mathrm{a}_{\mathrm{m} \nu^{\prime}}+\mathrm{b}_{\mathrm{m} \nu} \mathrm{b}_{\mathrm{m} \nu^{\prime}}\right) \mathrm{S}_{\nu \nu^{\prime}}^{\mathrm{m}}\left(\mathrm{r}_{\mathrm{i}}, \mathrm{r}_{0}\right)}{\sum_{\nu}\left(\mathrm{a}_{\mathrm{m} \nu}^{2}+\mathrm{b}_{\mathrm{m} \nu}^{2}\right)}
\end{array}
$$


where

$\mathrm{a}_{\mathrm{m} \nu}=\left(\mathrm{q}_{\mathrm{m} \nu}+\mathrm{q}_{-\mathrm{m} \nu}\right), \quad \mathrm{b}_{\mathrm{m} \nu}=\mathrm{i}\left(\mathrm{q}_{\mathrm{m} \nu}-\mathrm{q}_{-\mathrm{m} \nu}\right)$

and

$S_{\nu \nu^{\prime}}^{\mathrm{m}}\left(\mathrm{r}_{\mathrm{i}}, \mathrm{r}_{0}\right)=\int_{\mathrm{r}_{\mathrm{i}}}^{\mathrm{r}_{0}} \mathrm{rdr} \mathrm{R}_{\mathrm{m} \nu}(\mathrm{r}) \mathrm{R}_{\mathrm{m} \nu^{\prime}}(\mathrm{r})$

We define the 'energy distribution' (see Fig. 5) of this mode as the quantity

$F_{m}\left(r_{i}\right)=\lim _{r_{0}-r_{i}} \frac{\zeta_{m}\left(r_{i}, r_{0}\right)}{r_{0}-r_{i}}$

An analogous result to Eq. (A.13) can be written down for the fractional energy content of the projection component $\mathrm{g}_{\mathrm{m}}$, but with the advantage that the integral terms are independent of $m$. Fixing the fractional energy content of the image in some region of $D$ serves to constrain the recovered projection moments $p_{m}$ through the relation Eq. (A.2). We note that this reconstruction method is particularly useful in situations where some part of the projection data (for example from interior regions) is unavailable. Though computationally expensive (as for most non-linear iterative reconstruction schemes), it is nevertheless well suited for sparse data experiments where incorporation of prior knowledge is important.

The constrained minimization of the chi-squared measure (least squares) mollifies the ill-condition and allows reliable image reconstruction, as illustrated in Fig. 13(b) and (c). The recovered profiles are Abel inversions of the symmetric part of the equilibrium emission at a time slice before the disruption $\left(\mathrm{t}=41.0 \mathrm{~ms}\right.$ ) and for maximum radial orders $\nu_{\max }=20$, 24,28 and 32. The reconstruction bandwidth is $\sim \nu_{\max }$ radians per unit length [30] compared with the experimental Nyquist limit $\omega_{\max }=\pi / \Delta \mathrm{t} \sim 28$ radians per unit length, where $\Delta t \sim 0.11$ is the sampling rate on the unit circle. Notice that image energy constraints (imposed in the region $r>0.9$ ) suppress the spurious ringing artifacts and that the emission profiles vary little with $\nu_{\max }$ unless $\nu_{\max }$ exceeds the data bandwidth $\omega_{\max }$. The energy constraints thus allow the reconstruction bandwidth to approach the Nyquist limit without excessive sensitivity to noise. The SXR reconstructions presented in this work were limit to a maximum radial order $\nu_{\max }=24$.

\section{ACKNOWLEDGEMENTS}

The authors are indebted to the LT-4 team for providing the high quality experimental data. They are also grateful to R.D. Parker, P. Smeulders and J. Strachan for helpful discussions and would like to thank S.M. Hamberger and R.L. Dewar for carefully reading the manuscript and offering useful comments. Thanks are also due to B.D. Blackwell for his help with the colour graphics.

\section{REFERENCES}

[1] ALLADIO, F., BARDOTTI, G., BARTIROMO, R., et al., Nucl. Fusion 26 (1986) 11.

[2] NAGAMI, M., YOSHIDA, H., SHINYA, K., et al., Nucl. Fusion 22 (1982) 409.

[3] TOI, K., ITOH, S., KADOTA, K., et al., Nucl. Fusion 19 (1979) 1643.

[4] WESSON, J.A., GILL, R.D., HUGON, M., et al., Nucl, Fusion 29 (1989) 641.

[5] WESSON, J., GOWERS, C., HAN, W., et al., in Controlled Fusion and Plasma Physics (Proc. 12th Eur. Conf. Budapest, 1985), Vol. 9F, Part I, European Physical Society (1985) 147.

[6] JET Team (presented by P.H. Rebut), in Plasma Physics and Controlled Nuclear Fusion Research 1990 (Proc. 13th Int. Conf. Washington, DC, 1990), Vol. 1, IAEA, Vienna (1991) 27.

[7] GIBSON, A., Nucl. Fusion 16 (1976) 546.

[8] ASHBY, D.E.T.F., HUGHES, M.H., Nucl. Fusion 21 (1981) 911

[9] WHITE, R.B., MONTICELLO, D.A., ROSENBLUTH, M.N., Phys. Rev. Lett. 44 (1980) 1618

[10] PERSSON, M., BONDESON, A., in Theory of Fusion Plasmas (Proc. Workshop Varenna, 1987), Editrice Compositori, Bologna (1988) 325.

[11] CAMPBELL, D.J., DUPERREX, P.A., EDWARDS, A.W., et al., in Plasma Physics and Controlled Nuclear Fusion Research 1986 (Proc. 11th Int. Conf. Kyoto, 1986), Vol. 1, IAEA, Vienna (1987) 433.

[12] PERSSON, M., BONDESON, A., Nucl. Fusion 29 (1989) 989.

[13] BONDESON, A., PARKER, R.D., HUGON, M., SMEULDERS, P., Nucl. Fusion 31 (1991) 1695.

[14] KLEVA, R.G., DRAKE, J.F., DENTON, R.E., Comments Plasma Phys. Control. Fusion 13 (1989) 63; KLEVA, R.G., DRAKE, J.F., Phys. Fluids B 3 (1991) 372.

[15] BELL, M.G., CHEETHAM, A.D., HAMBERGER, S.M., et al., Aust. J. Phys. 37 (1984) 137.

[16] CHEETHAM, A.D., HAMBERGER, S.M., HOW, J.A., et al., Aust. J. Phys. 39 (1986) 35.

[17] GRANETZ, R.S., SMEULDERS, P., Nucl. Fusion 28 (1988) 457.

[18] SAUTHOFF, N.R., VON GOELER, S., STODIEK, W., Nucl. Fusion 18 (1978) 1445. 
[19] KLÜBER, O., ZOHM, H., BRUHNS, H., GERNHARDT, J., KALLENBACH, H., ZEHRFELD, H.P., Nucl. Fusion 31 (1991) 907.

[20] CORMACK, A.M., J. Appl. Phys. 35 (1964) 2908.

[21] EDWARDS, A.W., CAMPBELL, D.J., ENGELHARDT, w.W., et al., Phys. Rev. Lett. 57 (1986) 210

[22] TSUJI, S., NAGAYAMA, Y., MIYAMOTO, K., KAWAHATA, K., NODA, N., TANAHASHI, S., Nucl. Fusion 25 (1985) 305.

[23] MIRNOV, S.V., SEMENOV, I.B., in Plasma Physics and Controlled Nuclear Fusion Research 1976 (Proc. 6th Int. Conf. Berchtesgaden, 1976), Vol. 1, IAEA, Vienna (1977) 291.

[24] Hanada, K., OGURA, K., IDE, S., et al., Plasma Phys. Control. Fusion 32 (1990) 1289.

[25] JANICKI, C., DECOSTE, R., SIMN, C., Phys. Rev. Lett. 62 (1989) 3038
[26] WOLFE, S.W., EDWARDS, A.W., GILL, R.D., NAVE, M.F.F., WESSON, J.A., in Controlled Fusion and Plasma Heating (Proc. 17th Eur. Conf. Amsterdam, 1990), Vol. 14B, Part I, European Physical Society (1990) 335.

[27] WESSON, J.A., Plasma Phys. Control. Fusion 59 (1986) 2303.

[28] KLEVA, R.G., DRAKE, J.F., DENTON, R.E., Phys. Fluids 30 (1987) 2119.

[29] ROSENBLUTH, M.N., MONTICELLO, D.A., STRAUSS, H.R., WHITE, R.B., Phys. Fluids 19 (1976) 1987.

[30] HOWARD, J., Opt. Soc. Am., A 5 (1988) 999.

(Manuscript received 5 June 1991

Final manuscript received 11 October 1991) 